\title{
Automatisation d'un banc de mesures (application aux réseaux électrocinétiques et à l’algèbre linéaire)
}

\author{
P. LAGONOTTE
}

\author{
I.U.T. de Poitiers, Département GEII
}

\begin{abstract}
Résumé : Nous présentons dans ce document un projet traité en enseignement d'IUT deuxième année, et qui est relatif à la conception et à la mise en œuvre d’un banc de mesures informatisé. La partie logicielle est programmée sous LabVIEW, et la partie matérielle correspond à une centrale de mesure de type Agilent 34970A équipée d'une carte multiplexeur 20 voies (Agilent 34901A), et d'une carte de matrice de commutation 4x8 (Agilent 34904A). Une liaison par bus GPIB permet de relier les deux parties qui forment alors un banc de mesures informatisé. L’application pratique au contrôle de la valeur des éléments d’un réseau de résistances est décrite aussi bien au niveau de la partie câblée que de la partie programmée. L'ensemble de ce projet formant un sujet suffisamment simple, ambitieux et cohérent.
\end{abstract}

Mots Clés : algèbre linéaire, centrale de mesures, programmation LabVIEW, informatique industrielle, Bus GPIB/IEEE 488-2, traitement de l'information, projet d'étude et de réalisation.

\section{Introduction}

Dans le cadre d'un projet de deuxième année d’I.U.T. de génie électrique (option automatique), il nous a paru intéressant d'étudier l'automatisation d'un banc de mesures et le traitement possible des informations issues de mesures. Cette automatisation fait appel à des connaissances diverses et variées : programmation sous LabVIEW, utilisation de drivers d'instruments, Bus IEEE 488-2, fonctionnement d'une centrale d'acquisition et des différentes cartes pouvant y être associées, raccordement et câblage.

La durée d'un projet étant limitée à neuf séances de quatre heures, il n’est pas toujours facile de trouver un sujet suffisamment simple, ambitieux et cohérent. Le problème de la détermination des éléments d'un réseau de résistances s'y prête bien. C'est la mise en œuvre de ce banc de tests et de mesures que nous souhaitons ici vous présenter.

Sur les neuf séances du projet quatre sont consacrées à une initiation à LabVIEW à l'aide d'exercices : manipulation des chaînes de caractères, manipulation des nombres (booléens, entiers, réels, complexes), manipulation des tableaux (matrices) et clusters (groupement de données), structures (condition, séquence, for, while), et l'affichage de mesures ou de résultats sous forme graphique.

Une séance est consacrée à la résolution mathématique du problème de la détermination des éléments du réseau de résistances, présentée dans [1]. Les mesures sont effectuées de manière manuelle, l'inversion matricielle est dans un premier temps effectuée à l'aide de calculatrices, et dans un second temps programmée sous LabVIEW.

Trois séances sont consacrées à l'utilisation de « drivers » d'instruments, au pilotage d'instruments par une liaison RS232 et GPIB, et à la mise en œuvre matérielle et logicielle du banc de mesures.

La dernière séance correspond à un contrôle individuel de projet de deux heures (en deux séries de six étudiants).

\section{La conception d'un banc de mesures}

Un banc de mesures est constitué des trois parties :

- des instruments de mesures contrôlables par une interface informatique, et qui effectuent l'acquisition de l'information ou la délivrance de signaux appropriés ;

- des communications (RS232, RS485, GPIB-488, USB, LAN,....) qui permettent de transmettre ou d'échanger des informations ;

- d'un programme informatique qui donne de manière séquentielle des ordres aux différents instruments, afin que ces derniers soit établissent des signaux soit renvoient les mesures effectuées, et qui effectue le traitement des diverses informations reçues. 
Le programme informatique a donc un rôle de chef d'orchestre entre les différents instruments intervenants, son rôle est fondamental dans le fonctionnement et la gestion de tels systèmes.

\subsection{Les aspects logiciel et programmation}

Actuellement différents langages de programmation peuvent être utilisés pour le contrôle d'un banc de mesures. En plus des classiques $\mathrm{C} / \mathrm{C}++$ et VisualBasic s'écrivant avec de classiques lignes de code, un certain nombre de langages ont été développés pour faciliter la gestion des entrées-sorties (RS232, RS 485, GPIB, USB, LAN, fichiers disque dur), ainsi que la mise au point et la gestion d'une interface graphique de type superviseur (écran, souris, clavier). Nous citerons entre autre TestPoint 4.0 [2] ; Agilent VEE PRO 7.0 [3] ; LabWindows 7.0 ; LabVIEW 7.0 [4] [5] . Ces logiciels ont été développés par des constructeurs d'instruments, respectivement Keithley, Agilent (anciennement Hewlett-Packard) et National Instruments pour des besoins de contrôle de leurs appareils.

\section{Présentation et initiation à LabVIEW}

LabVIEW est un logiciel qui permet de configurer des instruments, piloter des manipulations, recueillir et traiter des données. Toute application LabVIEW est appelée un instrument virtuel (Virtual Instrument : VI). Pour chaque instrument, ou bus, ou carte d'acquisition, il existe une bibliothèque de programmes de configuration qui fournit la face avant de l'instrument et convertit les commandes et les mesures.

Un instrument de mesure classique peut se décomposer en quatre parties logiques :

- la première partie réalise la conversion d'une grandeur physique en signal électrique (exemple d'une thermistance et de son alimentation). C'est le conditionnement des signaux ;

- la seconde partie réalise la mise en forme de cette grandeur électrique quelconque en une grandeur électrique exploitable par des circuits électroniques ;

- la troisième partie est constituée des circuits électroniques de traitement et/ou d'analyse (exemple du calcul du spectre fréquentiel d'un signal) ;

- la quatrième partie réalise l'affichage des résultats (exemple du tube cathodique d'un oscilloscope, ou des afficheurs numériques d'un voltmètre) et permet de changer les paramètres de l'instrument (exemple du potentiomètre réglant l'échelle de visualisation sur l'écran de l'oscilloscope).

De même, un instrument de mesure informatique va se décomposer suivant quatre parties :

- une première partie identique à la précédente mais réalisée par des circuits spécifiques de mise en forme des grandeurs physiques en une tension exploitable (exemple : 0-5 V), c'est ce que font les cartes de conditionnement de signaux (SCXI, ...) ;

- la seconde partie convertit ces signaux électriques mis en forme en une grandeur numérique et stocke ces valeurs dans la mémoire de l'ordinateur (cartes de conversion analogiques/numériques) ;

- la troisième partie traite et analyse ces valeurs numériques, ce travail est réalisé par le logiciel ;

- la quatrième partie affiche les résultats sur l'écran de l'ordinateur, et permet de changer les paramètres de l'instrument grâce à la souris ou au clavier. Ce travail est également réalisé par le logiciel.

On appelle "instrument virtuel" l'ensemble composé des troisième et quatrième parties de l'instrument. Avec LabVIEW, on pourra manipuler ces instruments virtuels comme s'il s'agissait d'instruments réels : on pourra par exemple tourner un potentiomètre (virtuel puisque c'est une image de potentiomètre) grâce à la souris ou bien visualiser une courbe sur une portion de l'écran représentant un oscilloscope.

Le « Tutorial LabVIEW » en français fourni avec le logiciel [4] (en version pdf) est bien fait, permet un autoapprentissage et donne des idées pour une série d'exercices progressifs avec les étudiants. Une autre solution consiste à utiliser l'ouvrage de référence de Francis Cottet [5]. Faire un cours sur LabVIEW dépasserait toutefois le cadre de ce document. D'autres informations précieuses au sujet des différents bus d'instrumentation [6], du bus GPIB ou IEEE 488 [7] et des pilotes d'interfaces NI-VISA [8] sont disponibles sur le serveur de National Instrument.

\section{Le choix d'un langage de programmation}


Généralement le langage de programmation utilisé fait parti de la culture d'une entreprise, d'un établissement ou d'un laboratoire. Le choix d'un langage de programmation n'est en général plus à faire puisqu'il a été fait de manière très antérieure, et est généralement imposé pour tout nouveau projet. C’est également le cas au département G.E.I.I. de Poitiers, où LabVIEW avait été introduit en T.P. d'automatique par Dominique JACOB quelques années auparavant, et où le logiciel sous licence est disponible.

Le langage LabVIEW est très répandu dans l'industrie. Son succès vient du fait que :

- pour arriver à vendre ses instruments, un fabricant doit être en mesure de proposer des « drivers LabVIEW » correspondant à ses produits et permettant leur mise en œuvre facile et rapide par ses clients.

- National Instruments encourage les fabricants d'instruments à développer des « drivers LabVIEW » afin que LabVIEW puisse très facilement commander un ensemble d'appareils de marques hétérogènes.

Cependant la concurrence entre fabricants est sévère dans l'industrie de l'instrumentation. National Instruments propose gratuitement des drivers LabVIEW pour les appareils d'Agilent, alors qu'Agilent vend ses instruments avec des bibliothèques pour son logiciel Agilent VEE.

D'autre part, la compatibilité au niveau d'un PC entre un langage de programmation de haut niveau d'une marque et des cartes d'interface d'une autre marque n'est pas forcément assurée au niveau des bibliothèques de liens dynamiques (fichier.dll). Aussi, il est sage de rester dans un univers informatique dont la cohérence est validée par un même et unique fabricant.

Le choix de programmer avec LabVIEW apparaît être en phase avec les développements industriels actuels, et ce d'autant plus que nos étudiants n'ont pas eu l'occasion d'utiliser un langage de programmation graphique (Langage G).

\subsection{La centrale de mesures}

\section{La centrale de mesures Agilent 34970A}

La centrale de mesures 34970A (Figure 1) est devenue un classique de l'industrie du fait de son coût très abordable et de ses nombreuses possibilités [9]. Elle se compose d'une unité centrale à trois logements avec un multimètre numérique à 6 chiffres $1 \frac{1}{2}$ incorporé. Le multimètre numérique interne assure le conditionnement, l'amplification (ou l'atténuation) des signaux et leur conversion analogiquenumérique avec une résolution jusqu'à 22 bits.
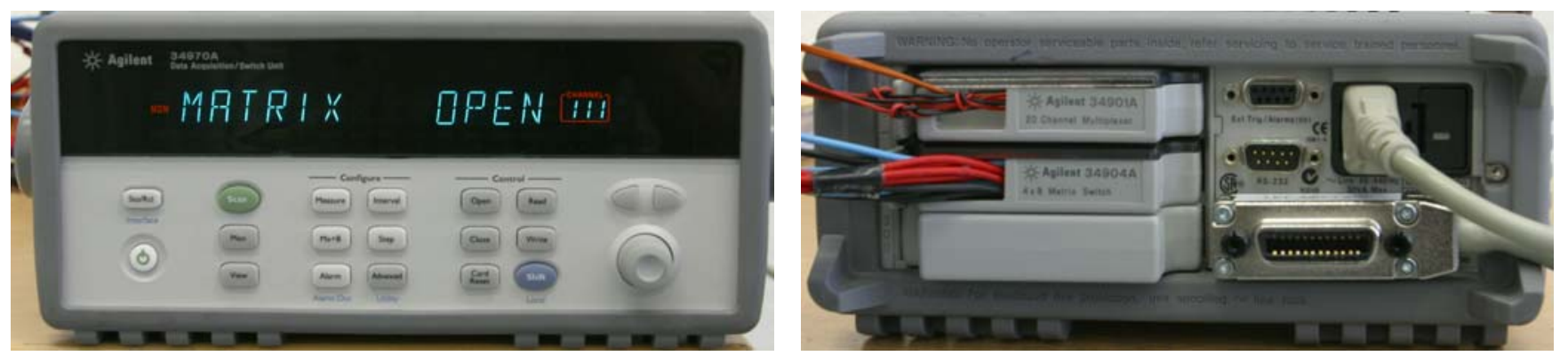

Fig. 1. Le châssis de la centrale de mesures Agilent 34970A (faces avant et arrière)

Les bornes de raccordement à vis sur les cartes suppriment l'usage de borniers extérieurs. De plus un dispositif compte toutes les fermetures des commutateurs afin d'assurer une maintenance fiable et prévisible des relais. Les entrées ou sorties d'une carte introduite dans le slot 1 sont numérotées à partir de l'adresse 100, à partir de l'adresse 200 pour le slot 2, et à partir de l'adresse 300 pour le slot 3 . Il est possible de choisir parmi huit modules enfichables pour créer un enregistreur de données compact, un système d'acquisition de données complet, une unité de commutation économique ou un automate de contrôle de processus industriels.

Les huit modules disponibles permettent de réaliser les fonctions suivantes :

- 34901A Carte multiplexeur à 20 voies (2/4 fils) Scrutation 60 voies/s. Des voies à deux et quatre fils peuvent être configurées sur le même module. Deux entrées supplémentaires protégées par fusible (22 
voies au total) acheminent un courant jusqu'à $1 \mathrm{~A}$ vers le multimètre numérique interne, ce qui permet des mesures de courant c.a. et c.c. sans recourir à des "shunts" (résistances en dérivation) externes.

- 34902A Carte multiplexeur à 16 voies (2/4 fils) Scrutation 250 voies/s. Le module 34902A emploie des relais "reed" pour obtenir des fréquences de balayage pouvant atteindre 250 voies par seconde pour la centrale Agilent 34970A. Ce module convient aux applications de tests automatisés à débit élevé, ainsi qu'aux tâches d'enregistrement et de surveillance des données à grande vitesse.

- 34903A Carte commutateur / Actionneur universel, 20 voies. Il peut être utilisé pour alimenter des équipements sous test, des voyants témoins ou actionner des relais externes et des solénoïdes. Associé à d'autres modules Agilent 3490xA de matrice et de multiplexeur, il permet de construire des systèmes de commutation personnalisés. Ses contacts $300 \mathrm{~V}, 1$ A peuvent accepter jusqu'à 50 watts, ce qui est suffisant pour de nombreuses applications de commutation de puissance.

- 34904A Carte de matrice 4 x 8. Des rangées ou colonnes de matrices peuvent être connectées entre 2 ou 3 modules Agilent pour construire des matrices $8 \times 8,4 \times 16$ ou plus, en ayant jusqu'à 96 points de croisement dans un seul châssis 34970A.

- 34905A Carte double multiplexeur RF 2 GHz 1:4 voies, 50 ohms. Il peut par exemple acheminer des signaux entre le dispositif à tester et les instruments tels que le générateur de signaux, oscilloscope, analyseur de spectre. Les multiplexeurs RF sont disposés en tant que deux multiplexeurs 1 x 4 indépendants, chacun ayant un blindage commun et un conducteur central commuté. Les connexions peuvent s'effectuer directement sur les entrées SMB avec une bande passante utilisable de $2 \mathrm{GHz}$ ou sur des adaptateurs BNCSMB fournis avec une bande passante de $1 \mathrm{GHz}$. Plusieurs groupes de voies peuvent être connectés en cascade pour des applications nécessitant des topologies encore plus grandes, pour créer un multiplexeur 16:1 sans adaptateur dans un même châssis.

- 34906A Carte double multiplexeur RF 2 GHz 1:4 voies, 75 ohms. Identique au 34905A mais en 75 ohms d'impédance.

- 34907A Carte multifonctions. Elle combine deux ports Entrées/Sorties numériques 8 bits, un totalisateur $100 \mathrm{kHz}$ permettant de compter le nombre de fronts montants ou descendants et deux sorties analogiques $\pm 12 \mathrm{~V}$ - le tout dans un seul module référencé à la terre. Les entrées numériques et l'entrée du totalisateur peuvent être incluses dans un balayage de voies. Les limites d'alarme sont évaluées en permanence pour les entrées numériques et du totalisateur, capturant et enregistrant les conditions d'alarme entre les scrutations.

- 34908A Carte multiplexeur 40 voies unipolaires. Chaque module commute 40 entrées, chaque entrée étant un seul fil. Toutes les mesures internes sur deux fils, à l'exception de l'intensité, sont supportées. Le point froid (masse commune) du module est isolé de la terre et peut flotter jusqu'à $300 \mathrm{~V}$.

\section{La carte multiplexeur à 20 voies : Agilent $34901 \mathrm{~A}$}

La carte 34901A est le multiplexeur polyvalent pour le balayage de voies par la centrale Agilent 34970A. Ce module est divisé en deux rangées de dix voies chacune pour différentes mesures à deux fils (Figures 2 et 3). Chaque voie peut être configurée indépendamment des autres sans avoir besoin d'un conditionnement spécifique du signal. Les différentes configurations de mesure possibles sont :

- température (thermocouple, RTD ou thermistance). Le module 34901A comporte un bloc isotherme intégré de référence de température pour réduire les erreurs lors de mesures par thermocouple ;

- tension (continue ou alternative jusqu'à $300 \mathrm{~V}$ ) ;

- courant (continu ou alternatif jusqu'à 1 A). Deux voies supplémentaires protégées par fusible (ce qui porte à 22 le nombre de voies au total) supportent un courant jusqu'à $1 \mathrm{~A}$ et sont reliées au multimètre numérique interne, ce qui permet des mesures de courants alternatif et continu sans recourir à des shunts externes. En cas de recours à un shunt (résistante de précision et de puissance) externe, l'appareil est configuré en millivoltmètre ;

- fréquence et période jusqu'à $300 \mathrm{kHz}$;

- résistance (sur deux ou quatre fils jusqu'à $100 \mathrm{M} \Omega$ ). Une mesure de résistance deux fils mesure en plus de la résistance de l'élément les résistances des fils nécessaires au câblage qui sont non négligeables dans le cas où l'élément à mesurer est de faible résistance. La méthode à quatre fils est utilisée pour mesurer des résistances de faible valeur dans toutes sortes d'applications, particulièrement pour les valeurs inférieures à $10 \Omega$, ainsi que dans les mesures qui nécessitent une 
grande précision, comme les mesures de résistance détectrice de température (RTD). Lorsque l'appareil est configuré en mesure de résistance à quatre fils, l'instrument apparie automatiquement les voies $\mathbf{n}$ en injection de courant et $\mathbf{n}+\mathbf{1 0}$ en mesure de tension. Des voies à deux et quatre fils peuvent être configurées sur le même module.

\section{Mesure d'une résistance à 2 fils}

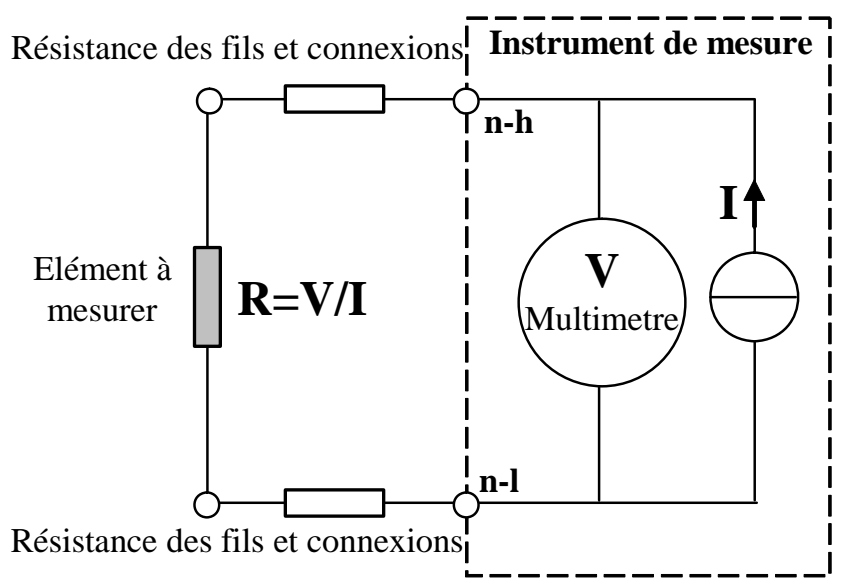

\section{Mesure d'une résistance à 4 fils}

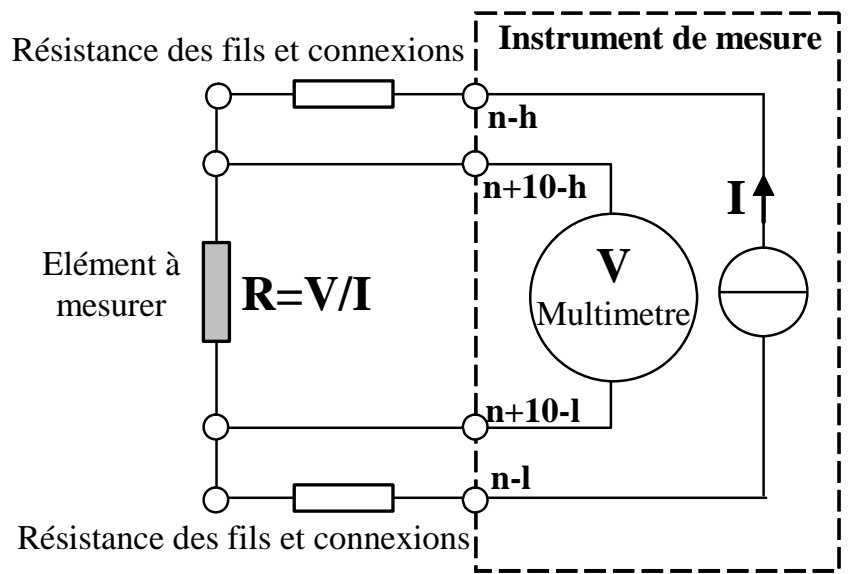

Fig. 2. Les mesures de résistances à deux fils et à quatre fils

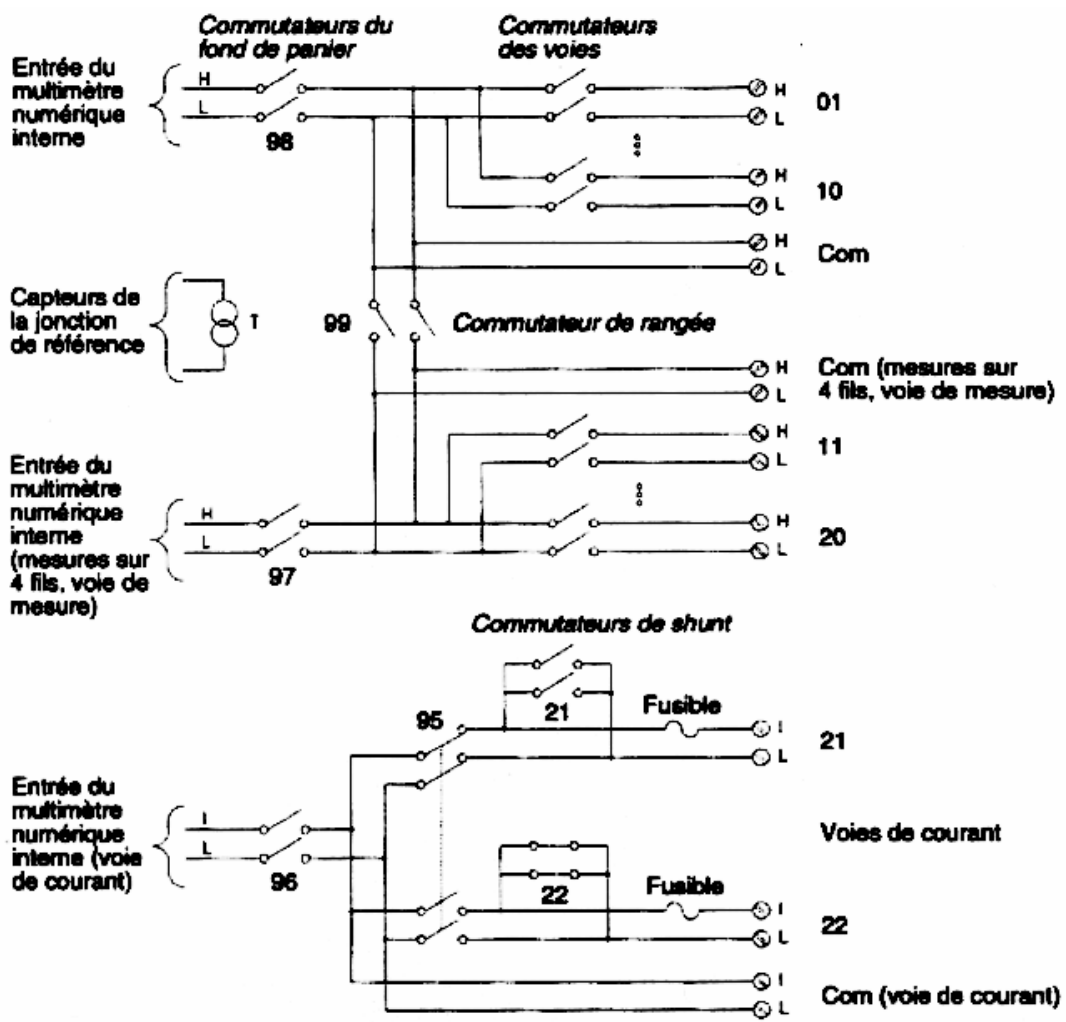

Fig. 3. Schéma de principe de fonctionnement de la carte 34901A

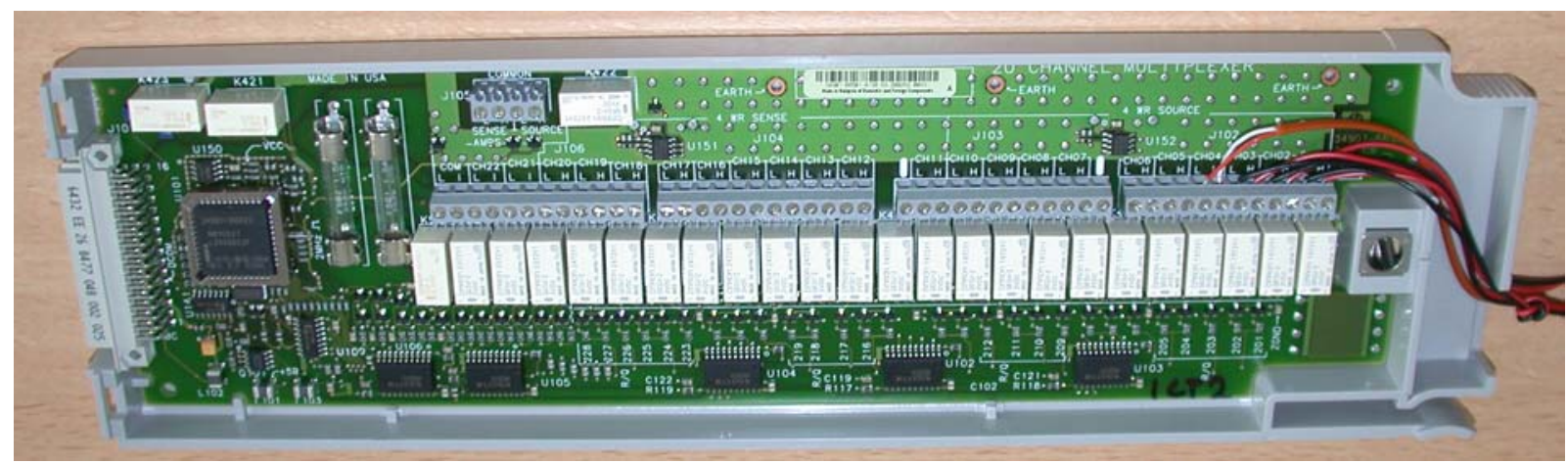

Fig. 4. La carte 34901A avec ses relais et ses borniers de câblage 


\section{La carte commutateur à matrice $4 \times 8$ : Agilent $34904 A$}

Le module 34904A contient 32 points de connexion pour deux fils disposés entre quatre lignes et huit colonnes d'accès (Figures 5 et 6). Il est alors possible d'effectuer toutes les combinaisons possibles entre une entrée et des sorties, ce qui permet à différents instruments d'être connectés en même temps à de multiples points sur un équipement sous test. Cette carte n’est pas raccordée au multimètre interne de la centrale 34970A.

Chaque relais de point de connexion est adressable individuellement pour une opération de fermeture ou d'ouverture. L'identification du relais se fait à partir des numéros de ligne et de colonne, par exemple la voie 41 correspond au relais de connexion de la quatrième ligne et de la première colonne.

Il est possible de fermer simultanément plusieurs voies d'un module, mais dans ce cas faire bien attention aux risques de court-circuit.

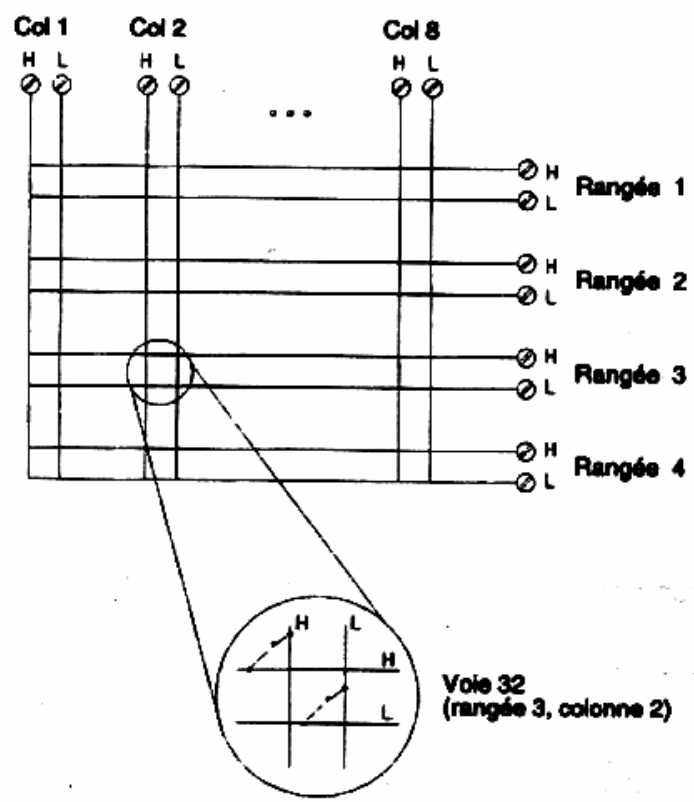

Fig. 5. Schéma de principe de fonctionnement de la carte 34904A

Des lignes ou colonnes de matrices peuvent être connectées entre 2 ou 3 modules pour construire des matrices 8x8, 4x16 ou plus, en ayant jusqu'à 96 points de croisement dans un seul châssis 34970A.

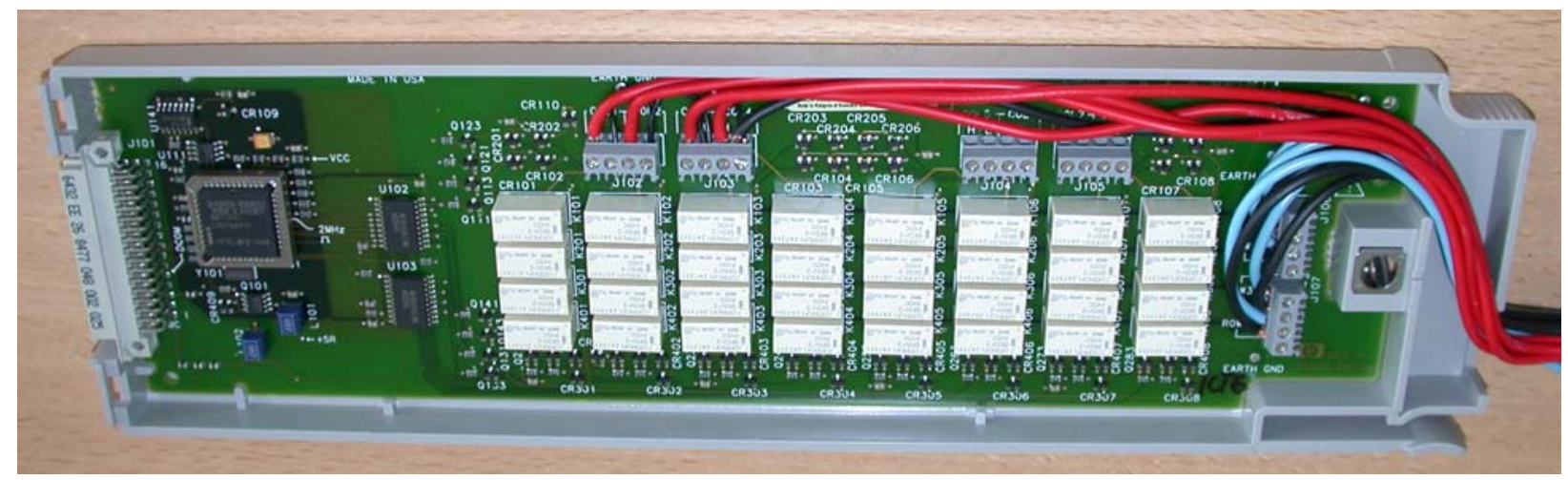

Fig. 6. La carte 34904A avec ses relais et ses borniers de câblage

\subsection{Les principales difficultés}

Une erreur généralement commise par les étudiants est d'oublier que l’information circulant sur les liaisons est identique à celle de l'électronique (analogique ou numérique 2 états). Si l'on veut éviter tout conflit, il faut toujours et obligatoirement avoir un seul et unique générateur au niveau d'une liaison (figure 7). 


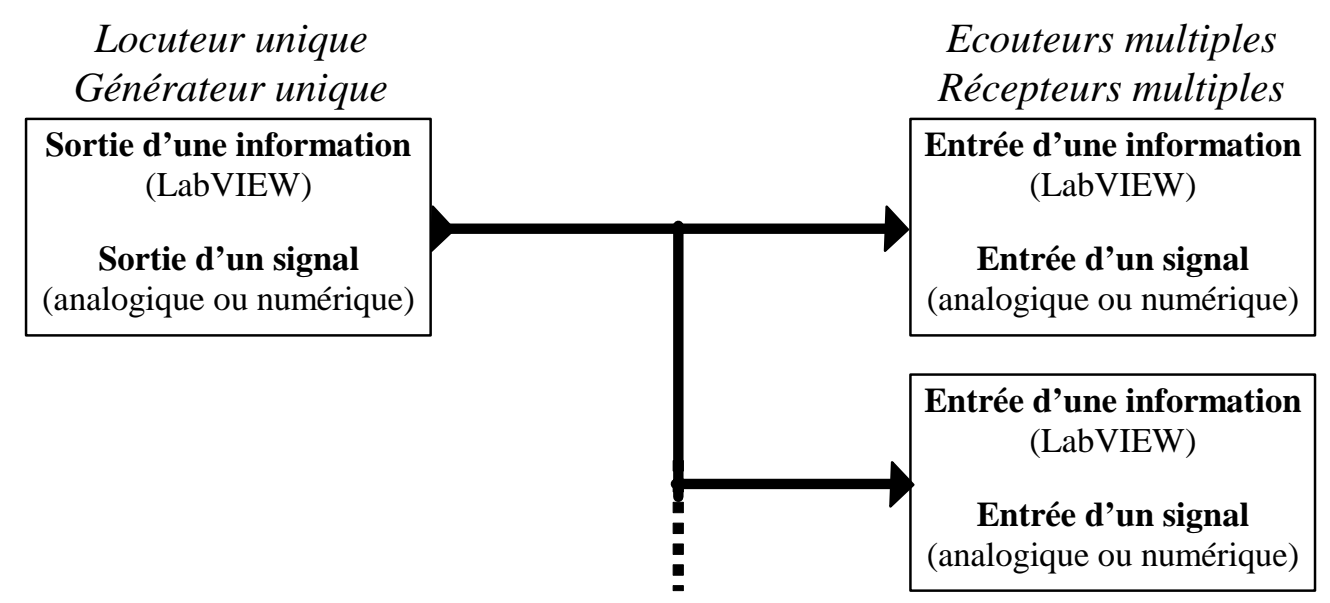

Fig. 7. Système mono-source et multi-récepteurs

Une autre difficulté concerne le passage de l'information dans les structures à l'aide de variables locales de séquence. La figure 8 présente le passage de l'information du bus d'adresse et du bus d'erreur aux sous VI contenues dans les différentes étapes d'une structure séquence. Nous pouvons remarquer la différence de câblage entre la première séquence, la dernière séquence et les séquences intermédiaires.

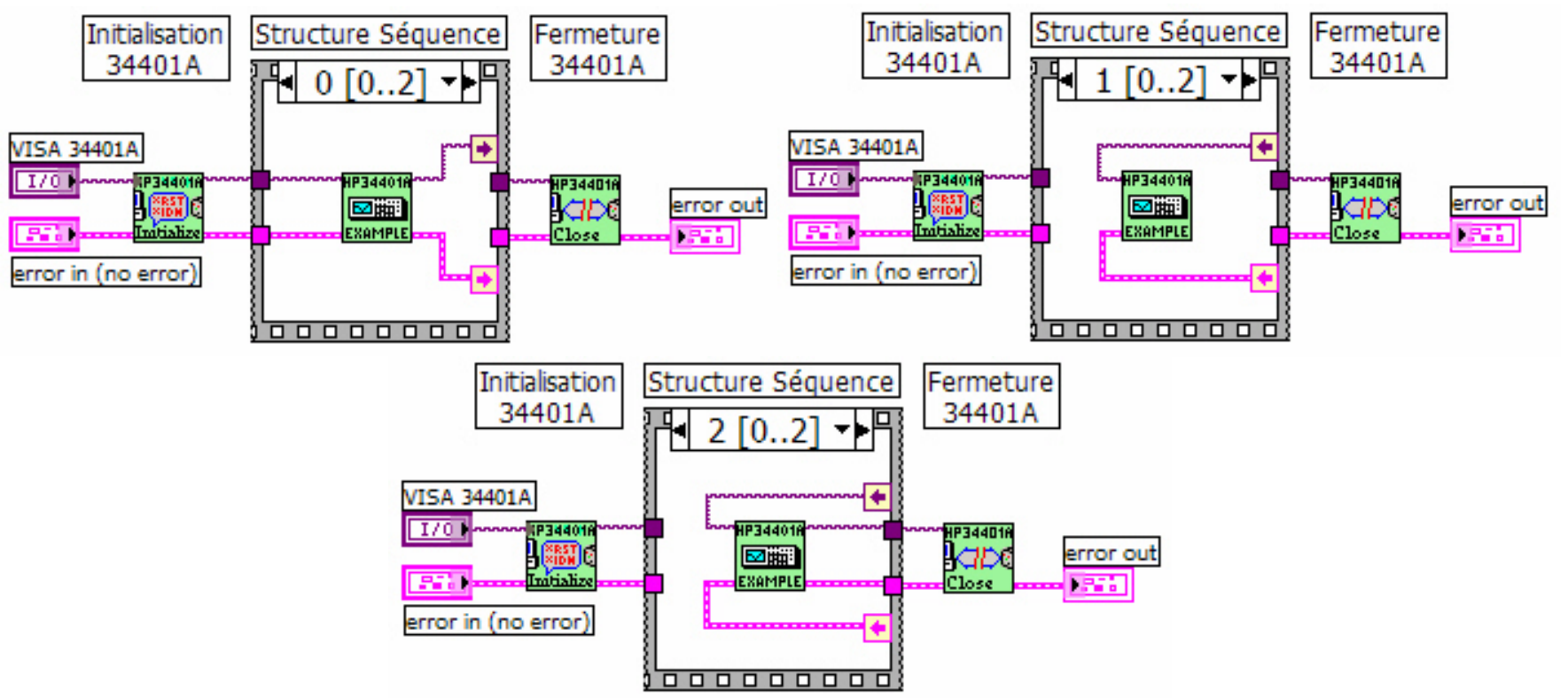

Fig. 8. L'utilisation de variables locales de séquence

Enfin une dernière difficulté concerne en cas de commande de plusieurs appareils interfacés, l'utilisation de plusieurs bus d'adresse (un par appareil de mesure) avec un seul bus d'erreur. La structure de principe du diagramme est présentée figure 9.

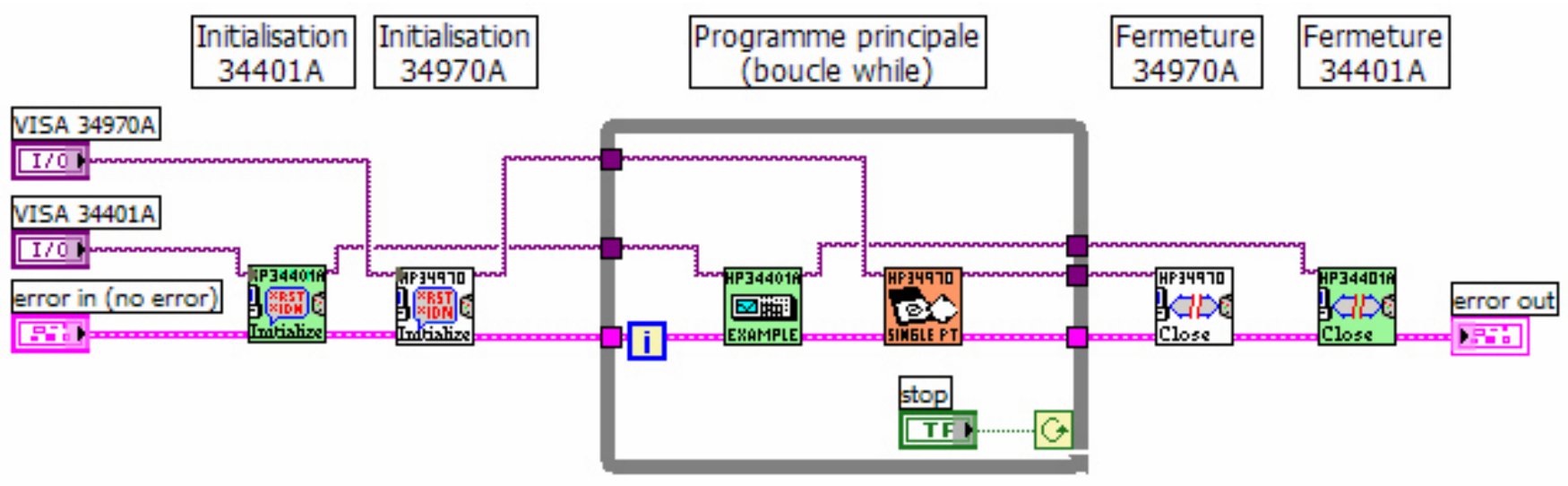

Fig. 9. Structure d'un programme multi instruments 


\section{Application pratique}

\subsection{Rappel sur l'objectif de l'application}

Pour mettre en pratique les notions de programmation d'une centrale de mesure sous LabVIEW, nous présentons ici un projet facile à mettre en œuvre. L'objectif est de déterminer les valeurs d'un ensemble de résistances sans avoir la possibilité d'accéder individuellement à chaque élément. Cette situation se présente concrètement lorsque des résistances ont été assemblées sur un circuit imprimé, et que l'on souhaite effectuer un test ou une vérification des composants en fin d'assemblage.

Les notions théoriques relatives à un réseau électrocinétique maillé qui permettent à partir de mesures d'impédances d'entrée et d'atténuation de déterminer la valeur de chaque composant sans avoir à déconnecter chaque élément du montage pour effectuer une mesure individuelle sont présentées dans une précédente partie "Réseaux électrocinétiques et algèbre linéaire (notions fondamentales) » [1].

Pour simuler et concrétiser cette situation, nous disposons d'une boîte Plexo comportant un réseau de résistances dont les accès sont reliés à des bornes de connexion vers l'extérieur (Figure 10).

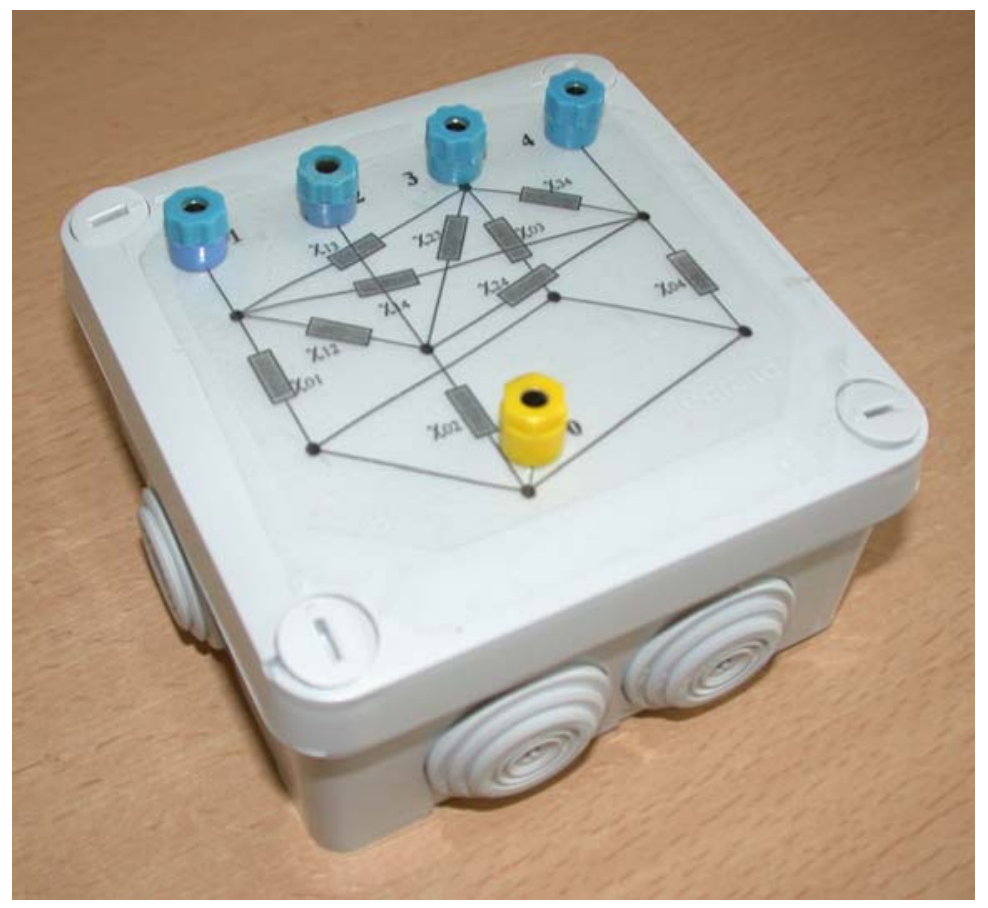

Fig. 10. La boîte Plexo et les bornes d'accès au réseau

La topologie de ce réseau à quatre nœuds correspond à celle de la figure 11.

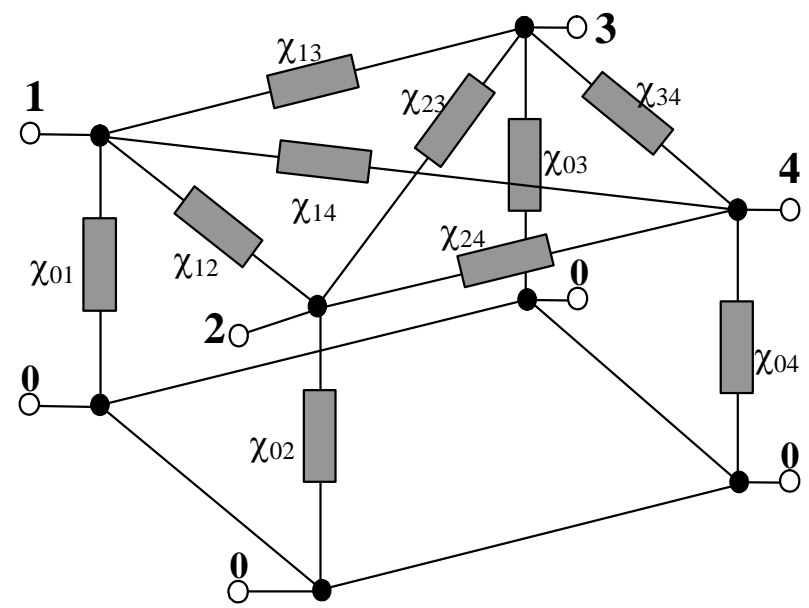

Fig. 11. Le réseau maillé de conductances considéré

L’objectif est de déterminer les valeurs des dix résistances constituant le réseau à partir de simples mesures au niveau des bornes d'accès. Les résistances ne sont pas accessibles individuellement pour effectuer des mesures à l'ohmmètre. Cependant, il est possible de mesurer la résistance d'entrée entre 
chaque nœud du réseau et le nœud de référence. D’autre part, en appliquant une tension entre un nœud du réseau et le nœud de référence, il est possible de mesurer l'atténuation de cette tension aux autres nœuds.

L’ensemble des mesures nécessaires à la détermination de la valeur de toutes les résistances peut être effectué de manière manuelle comme lors de travaux pratiques d'étudiants. La matrice des conductances de ce réseau à 4 nœuds est de dimension 4x4, soit 16 éléments. Mais comme cette matrice est symétrique, il n’y a en réalité que 10 inconnues à déterminer, soit le même nombre que le nombre de résistances à calculer. Cependant, lors d'un processus de fabrication en milieu industriel les mesures et leur traitement sont généralement automatisés. C’est cette automatisation qui fait l’objet de ce projet.

\subsection{Mise en ouvre matérielle}

Les centrales de mesures sont très utilisées dans l'industrie et dans les laboratoires de recherche, mais beaucoup plus rarement lors de classiques enseignements en EEA. Ce projet est l'occasion pour les étudiants de découvrir ce nouveau type de matériel. Dans le banc de test que nous avons développé (Figure 12), la centrale de mesures Agilent 34970A occupe une place centrale entre le PC et le montage que nous retrouverons au niveau du câblage et de la liaison informatique.

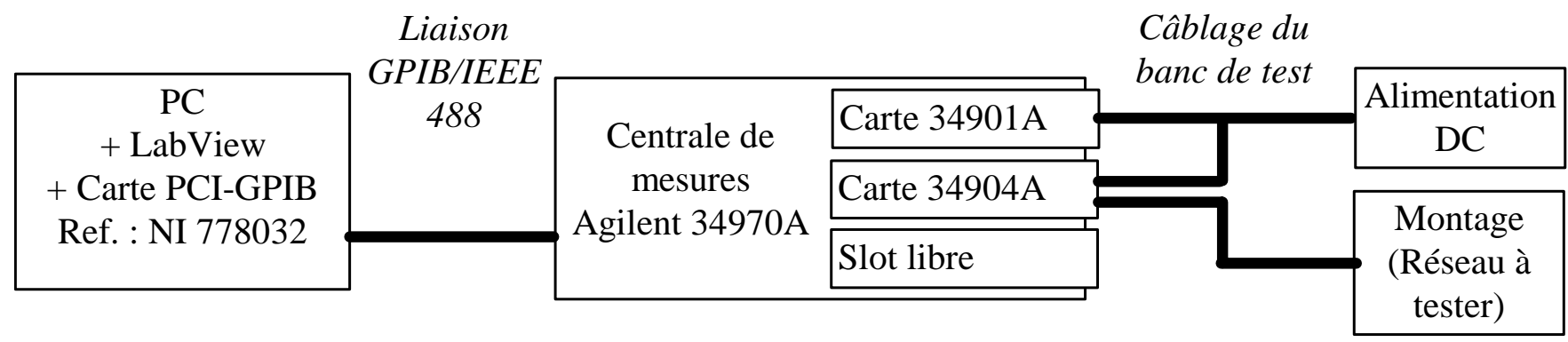

Fig. 12. Synoptique du banc de test développé

Nous devons effectuer la mesure de la résistance d'entrée par rapport au nœud de référence successivement aux quatre nœuds du réseau.

Nous devons pouvoir appliquer une tension successivement à chaque nœud du réseau et mesurer les atténuations de cette tension en tous les autres nœuds. La matrice de commutation permet d'appliquer la tension de l'alimentation à successivement tous les nœuds du réseau.

Disposant de 20 voies sur la carte 34901A, nous souhaitons éviter toute reconfiguration de voies lors du processus de mesure. Nous souhaitons également minimiser le câblage à effectuer, et qui est généralement la principale source de défaillance des systèmes. La figure 13 présente le câblage effectué et le choix de la configuration des différentes voies, lignes et colonnes.

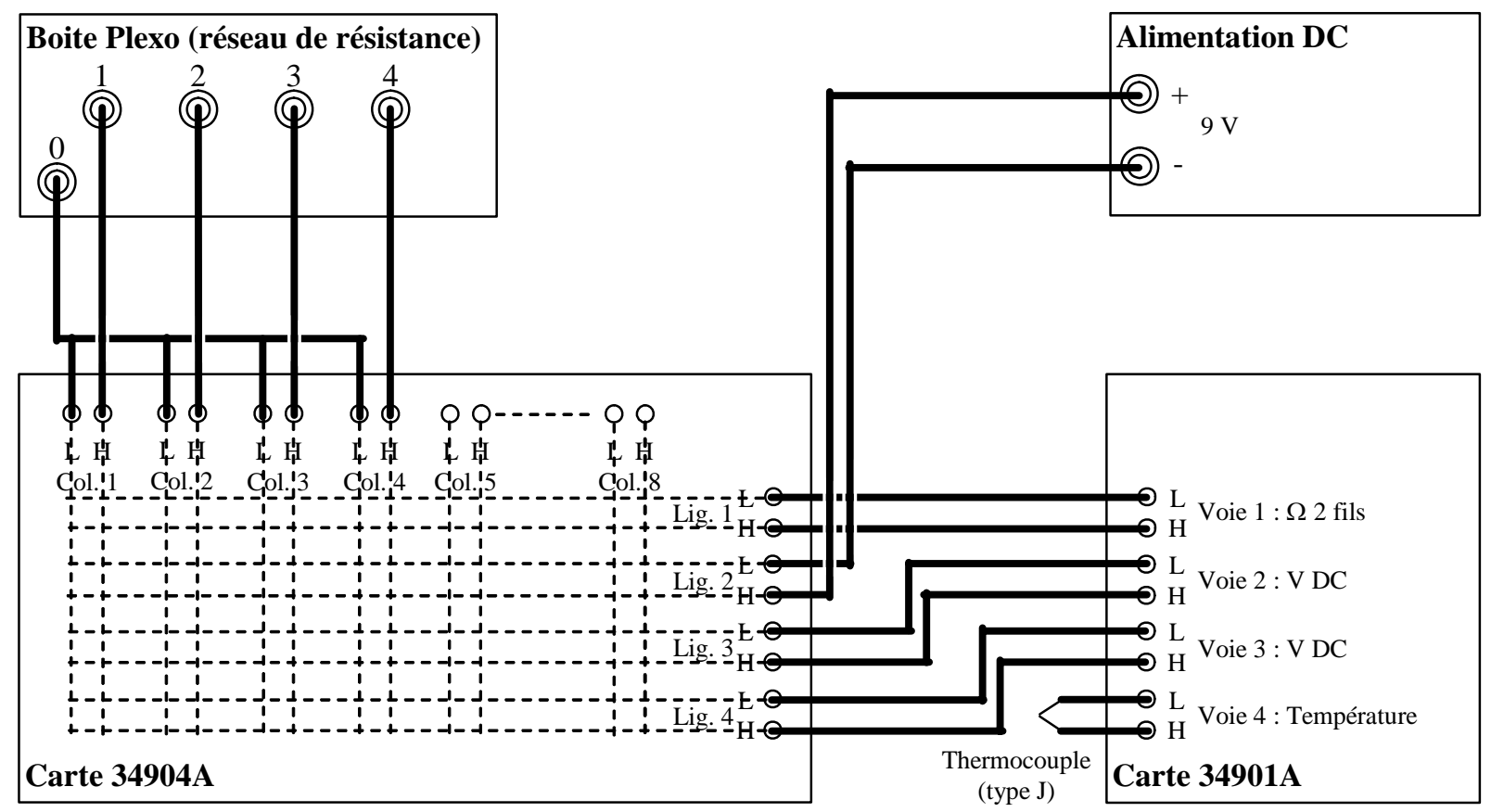

Fig. 13. Le schéma de câblage entre les cartes 34901A et 34904A, l'alimentation et la boîte Plexo 
Le déroulement des différentes mesures est alors le suivant :

Pour $\boldsymbol{i}=1$ à 4 :

- Fermeture du relais $n^{\circ} 10+\boldsymbol{i}$, carte $34904 A$.

- Mesure de l'impédance d'entrée au noud i mesure résistance $\left(r_{i i}\right)$, voie $n^{\circ} 1$ carte 34901A.

- Ouverture du relais $n^{\circ} 10+i$, carte 34904A.

- Fermeture du relais $n^{\circ} 20+\boldsymbol{i}$, (application d'une tension continue de $9 \mathrm{~V}$ entre les nœuds $\boldsymbol{i}$ et 0 ).

- Fermeture du relais $n^{\circ} 30+i$, carte $34904 A$.

- Mesure de la tension au nœud i mesure tension $\left(\Delta V_{i}\right)$, voie $n^{\circ} 2$ carte $34901 A$.

Pour $\mathbf{j}=1$ à 4 :

- Fermeture du relais $n^{\circ} 40+\boldsymbol{j}$, carte $34904 A$.

- Mesure de la tension au nœud $\mathbf{j}$ mesure tension $\left(\Delta V_{j}\right)$, voie $n^{\circ} 3$ carte 34901A.

- Calcul du terme $R_{i j}$ de la matrice, soit : $R_{i j}=r_{i i} . \Delta V_{j} / \Delta V_{i}$.

- Ouverture du relais $n^{\circ} 40+j$, carte 34904A.

Fin boucle $\mathbf{j}$.

- Ouverture du relais $n^{\circ} 30+i$, carte $34904 A$.

Fin boucle $\mathbf{i}$.

Le diagramme du sous-programme LabVIEW correspondant à cette séquence de mesures est présenté figure 16. La position des cartes dans les slots de la centrale est paramétrable.

Une mesure de la température peut être facilement effectuée au niveau de la voie $n^{\circ} 4$ carte 34901, afin de garder une trace des conditions de mesure.

Notons que si les cartes et le câblage sont dédiés à un banc spécifique de mesures, la centrale peut très facilement se déplacer entre différents bancs par le simple désenfichage des cartes.

Notons également la très bonne documentation en français de la centrale Agilent 34970A [9], et soulignons le grand intérêt pratique de la partie 8 de ce document consacrée aux « Techniques de mesure ».

\subsection{Mise en ouvre logicielle}

La figure 14 présente la face avant réalisée sous LabVIEW. Les matrices intermédiaires de calcul sont présentées afin de vérifier le bon fonctionnement du sous programme de mesure. La matrice [R] doit en effet être positive, symétrique et avoir une diagonale prépondérante.

Le programme est paramétrable au niveau de la liaison (RS232 ou GPIB), ainsi que le nombre de nœuds du réseau maillé. Les cartes 34901A et 34904A sont également interchangeables au niveau des 3 slots de la centrale 34970A et leur position paramétrable au niveau du programme. 


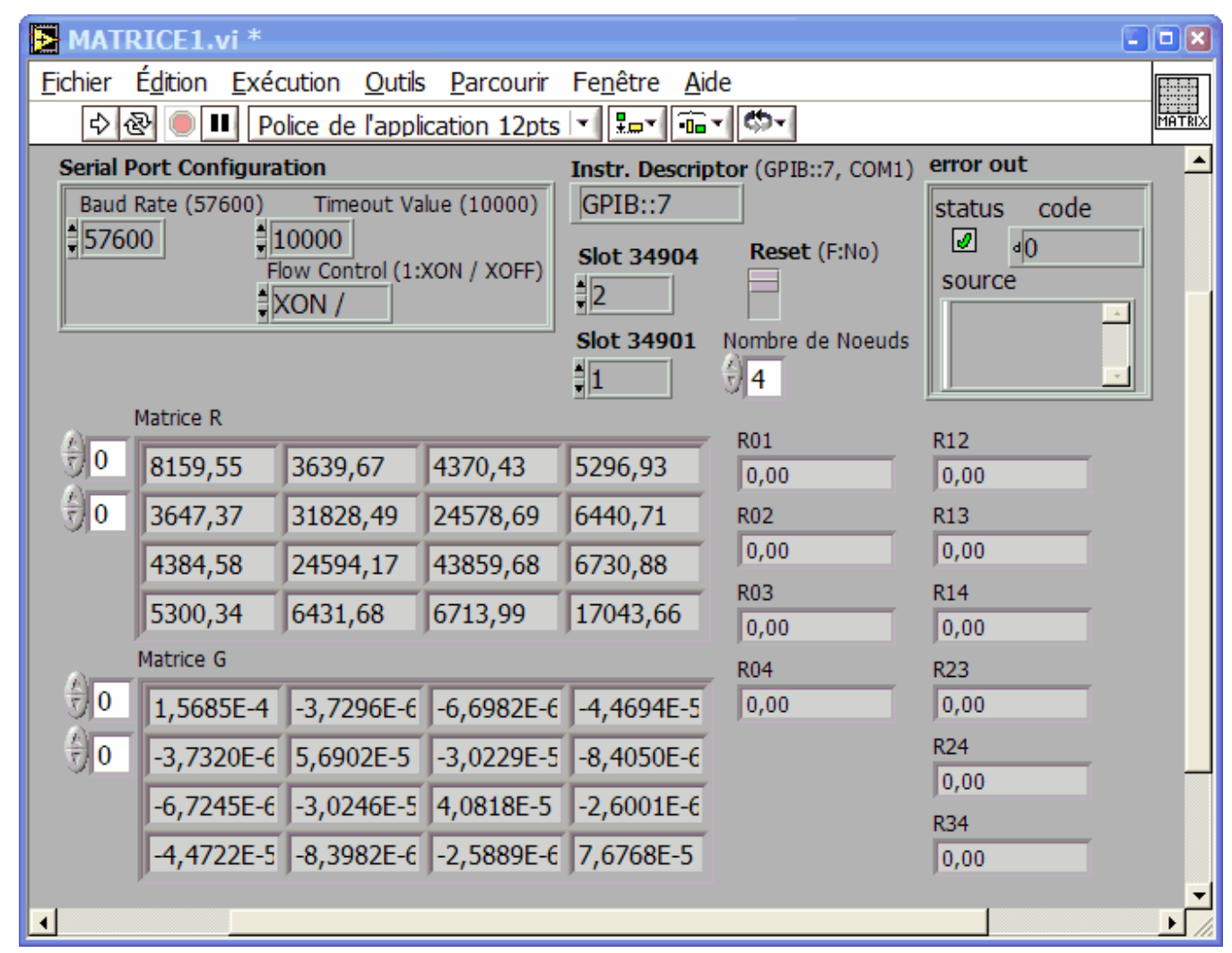

Fig. 14. La face avant du programme sous LabVIEW

La figure 15 présente le diagramme du programme principal. L’inversion matricielle est directement réalisée par un sous programme disponible avec la version LabVIEW 6. utilisée.

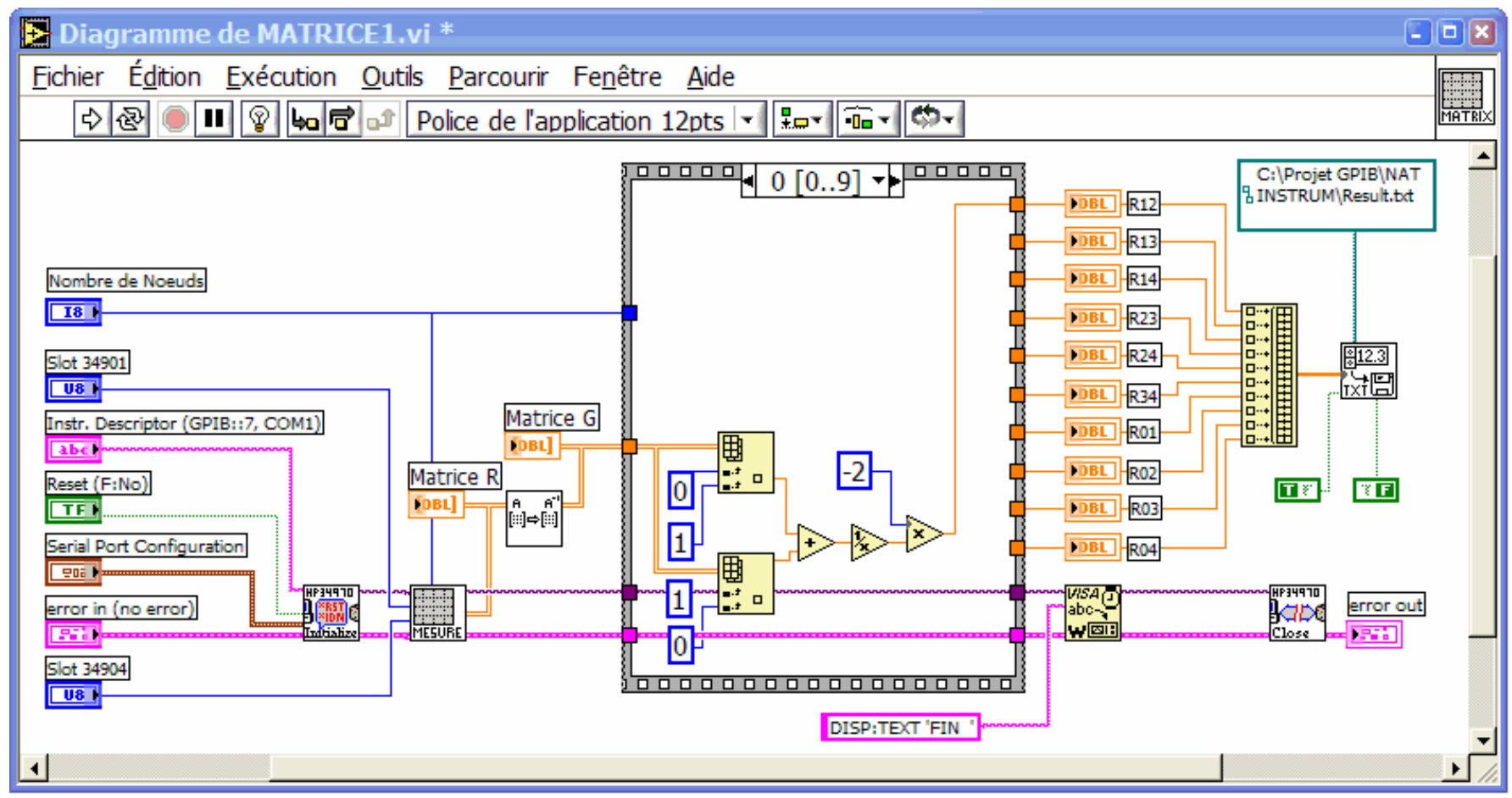

Fig. 15. Le diagramme du programme principal sous LabVIEW

La figure 16 présente le diagramme du sous-programme "Mesure Matrix » effectuant toutes les mesures et construisant la matrice des résistances [R]. Il est nécessaire de fermer les relais de la carte commutateur à matrice avant toute mesure et de les ouvrir après. 


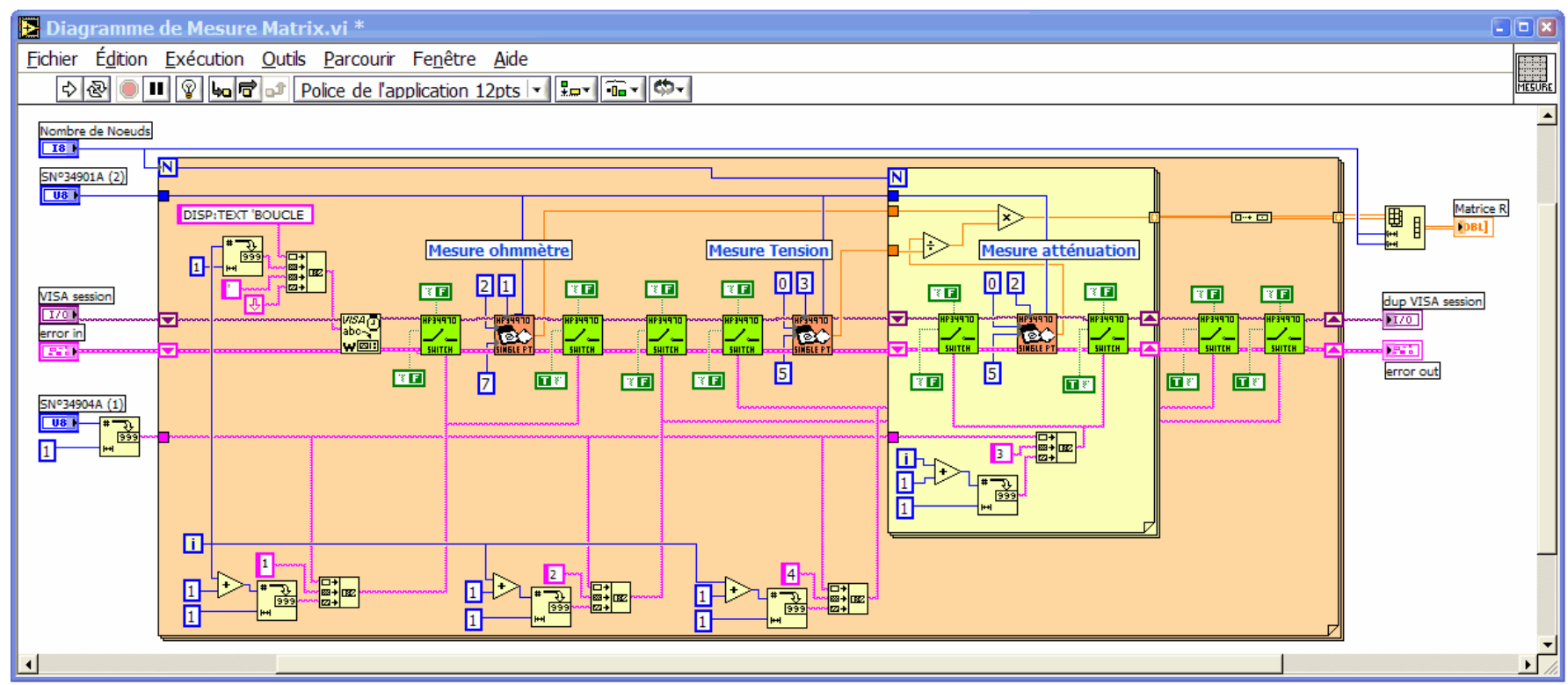

Fig. 16. Le diagramme du sous-programme « Mesure Matrix»

Le programme est constitué par deux boucles «for » imbriquées, la première effectue la mesure de l'impédance d'entrée aux quatre nœuds du réseau, ainsi que la mesure de la tension appliquée. La seconde boucle mesure la tension résultante aux autres nœuds et calcule les éléments de la matrice de résistances [R].

Nous avons essayé de rendre ce programme paramétrable au niveau du nombre de nœuds du réseau et de l'implantation des cartes 34901A et 34904A dans les différents slots de la centrale 34970A.

Il va de soit qu'un programme n'est jamais fini, car il peut toujours être complété par des fonctionnalités nouvelles comme la sauvegarde sur fichier des résultats avec la date et l'heure de la mesure, ainsi que par exemple la température ambiante.

\section{Conclusion}

Ce sujet de projet est très simple en apparence, mais il est en fait très formateur, car il fait appel à des connaissances diverses et variées devant se combiner pour arriver à un système opérationnel.

Cette étude permet une première approche des systèmes linéaires maillés, tout en ne faisant appel qu'à des notions supposées connues. Les notions fondamentales contenues dans [1], sont présentées aux étudiants lors de ce projet pour effectuer le traitement de l’information après les mesures.

Ce projet permet également de découvrir l'utilisation d'appareils de mesure au travers d'un bus d'instrumentation et de sa programmation. Ce projet très concret permet d'effectuer une initiation à la programmation sous LabVIEW d'instruments de mesure à l'aide de drivers développés pour la mise en œuvre rapide de systèmes industriels.

Un système d'instrumentation est souvent réalisé par l'assemblage de différents matériels de marques hétérogènes, mais dont le fonctionnement est parfaitement orchestré au niveau informatique, est une réalité industrielle. Le choix d'un instrument de mesure n’est plus uniquement lié à sa précision et à son coût, mais il est de plus en plus lié à son interfaçage et aux logiciels pouvant faciliter sa mise en œuvre.

\section{Références}

[1] P. LAGONOTTE, Y. EICHENLAUB, Réseaux électrocinétiques et algèbre linéaire (Notions Fondamentales), Revue J3EA.

[2] Site TestPoint : http://www.test-point.com

[3] Agilent VEE Pro http://we.home.agilent.com/FRfre/nav/-12247.536883294/pd.html Agilent VEE Pro User's guide http://cp.literature.agilent.com/litweb/pdf/E2120-90011.pdf et Agilent VEE Pro Advanced programming techniques http://cp.literature.agilent.com/litweb/pdf/E2120-90002.pdf 
[4] LabVIEW, Manuel de l'utilisateur, Édition de Janvier 2002, Référence 321190D-01 (au format .pdf et livré avec les CD de LabVIEW)

[5] F. COTTET, LabVIEW programmation et applications, Collection EEA série automatisme, ed. DUNOD.

[6] National Instrument, «L'utilisation des nouveaux bus dans le Test et la Mesure » http://digital.ni.com/worldwide/france.nsf/main?readform

[7] National Instrument, Le GPIB (IEEE 488) http://www.ni.com/products/gpib/f/

[8] National Instrument, NI-VISA : le standard de l'instrumentation sous LabVIEW http://digital.ni.com/worldwide/france.nsf/main?readform

[9] AGILENT TECHNOLOGIES, Guide d'utilisation Agilent 34970A Unité d'acquisition de données/commutation.

\section{Biographie de l'auteur :}

\section{Patrick LAGONOTTE}

I.U.T. de Poitiers, Département HSE, 8 rue Archimède, 79000 Niort

Laboratoire d’Etudes Thermiques, UMR CNRS n6608, ENSMA, BP 40109, 86961 Futuroscope Cedex, France.

Tel : 05-49-49-81-23 Fax : 05-49-49-81-01 Email : lagonotte@let.ensma.fr

Ancien élève de l'Ecole Normale Supérieure de Cachan, professeur agrégé de Génie Electrique en 1984, Docteur de l’Institut National Polytechnique de Grenoble en 1987. Il est Maître de Conférences à l'université de Poitiers depuis 1988 où il enseigne l'électrotechnique et est auteur d'un ouvrage sur les installations électriques. Il effectue ses recherches au Laboratoire d'Etudes Thermiques de l'ENSMA de Poitiers. Ses principaux domaines de recherche sont la modélisation des systèmes, la thermique des machines électriques, le refroidissement des semiconducteurs de puissance, les équations de diffusion et de propagation, la modélisation des systèmes d'ordre non entier ou d’ordre infini, la caractérisation des systèmes électrochimiques. 